\title{
Design of an Individual Pitch Controller for Offshore Wind Turbines Based on Neuro- adaptive Control
}

\author{
Lei Wang ${ }^{1,2}$, Lei Zhang ${ }^{1}$, Jianbo $\mathrm{Ke}^{1}$, Zeng Fan ${ }^{1}$, Jiawei Chen ${ }^{1,2}$, Wenxian Yang ${ }^{3}$ \\ ${ }^{1}$ First Department, College of Automation, Chongqing University, Chongqing, China \\ ${ }^{2}$ Second Department, State Key Laboratory of Power Transmission Equipment \& System Security and New Technology, \\ Chongqing University, Chongqing, China \\ ${ }^{3}$ Third Department, School of Engineering, Newcastle University, Newcastle upon Tyne NE1 7RU, UK.
}

\begin{abstract}
For large offshore wind turbines (WTs), pitch control is usually used for regulating generated power to the rated value and for mitigating the dynamic loads that at the wind speeds above the rated speeds. However, tracking the pitch angle accurately and quickly can hardly be realized due to complex operating environments, uncertain system parameters, various disturbances, and coupled effect between wind, wave, and turbine structure. In this paper, an individual pitch control system based on a neural adaptive strategy is proposed to address the problems related to uncertain system parameters and various disturbances. The proposed control method can achieve zero error tracking for the pitch angle in a predefined finite time. The design and stability analysis for the proposed method is elaborated. A simulation model is established in Matlab/Simulink, and by comparing it with the traditional PID control method, the merit of the proposed control scheme is verified.
\end{abstract}

\section{Introduction}

Wind Energy has been developed rapidly in the last decades and is already playing a role as a mainstream source of electricity[1-2]. In recent years, the research on large offshore floating WTs has become one of the important issues in the wind industry as floating WTs can harvest more reliable wind energy in deep water areas[3]. However, there exist grand challenges in the research and development of floating WTs.

For a large-scale offshore floating wind turbine(WT), it is typically required that the generated power should be limited to its rated value at above the rated wind speed to guarantee its safe operation[4-6]. Besides, the dynamic loads should be mitigated to prolong the service life of the WT. Such goals can be achieved by adjusting the pitch angle. However, the pitch control strategy for the floating WT may be quite complicated as the pitch system is a complex and fast-changing non-linear dynamic system, which has uncertain and changing system parameters and various disturbances [7]. So far, numerous control strategies have been deemed promising solutions for developing a pitch control system. Most of these strategies are traditional control algorithms, such as proportion integration differentiation control [8], disturbance accommodating control [9], and fullstate feedback control [10]. These traditional control algorithms are usually designed for one fixed operating point, and the controller parameters cannot be self-regulated; Besides, these control methods cannot deliver excellent performance in a non-linear time-varying WT system [11]. Advanced control strategies have also been deemed promising solutions [12]- [22].

Currently, individual pitch control (IPC) is usually adopted for offshore floating WTs, as it can help mitigate the dynamic loads caused by wind shear, tower shadow, wave effects, etc. So far, lots of advanced IPC strategies have been proposed. Navalkar et al. combines repetitive control (RC) with system identification for the IPC of WTs, which enables the IPC system to start from zero knowledge of the system dynamics and operating conditions, and be able to converge to a steady-state with high reductions in blade loads [23]. However, the algorithm is too complicated to be implemented in a real application. Zafer et al. propose a fuzzy logic controller based IPC method. Due to the use of FLC, the pitch control strategy does not rely on a detailed mathematical model of the WT's pitch system. However, the effectiveness of this strategy highly depends on the use of fuzzy rules and membership functions, which may be very hard to obtain [24]. In [25], a pitch regulation system is proposed, based on an advanced proportional and integral (PI) controller, whose proportional and integral coefficients are adjusted by a radial basis function (RBF) neural network (NN). Although effective, the proposed RBF based PI method is not able to deal with the problems related to unknown and time-varying system parameters. Mahmood et al. proposed a modelpredictive-control (MPC) based IPC system for WTs in [26]. It combines LIDAR measurement with MPC to effectively reduce unbalanced loads on the turbine blades. The problem of this method is that the LIDAR may work improperly due to errors in the measurement of wind speed. Besides, IPC methods based on the generic algorithm are also widely seen in literature [27]. However, it should be noted that timevarying uncertain system parameters and various disturbances are not adequately considered in the design of these IPC methods. Moreover, the tracking of the pitch angle in the predefined finite-time cannot be achieved. In all, these methods can hardly guarantee the performance of pitch angle regulation.

The main contribution of this paper is to propose an advanced IPC method to deal with the pitch angle tracking problem of offshore floating WTs in a finite and predefined time, on the condition that both time-varying uncertain 
system parameters and various disturbances exist. The rest of this paper is organized as follows: In Section 2, a non-linear dynamic model of the pitch system with uncertain parameters and unknown load disturbances is established. In Section 3, an individual pitch neural adaptive tracking controller with finite time tracking ability is proposed. Then, the proof of the system stability is elaborated. In Section 4, a simulation model is developed in Matlab/Simulink. Through comparison with the PID control method, the simulation results verify the effectiveness of the control strategy. Finally, conclusions are made in Section 5.

\section{Modeling of the pitch system}

The mechanical schematic diagram of a single blade pitch mechanism of a WT is shown in Figure 1 [28]. In the figure, $\beta_{i}$ is the pitch angle for the ith blade; $T_{d r i}$ is the driving torque of the ith blade pitch adjustment; $T_{t w i}$ is the sum of the torsional torque of the ith blade and the disturbance torque generated by the aerodynamic force, including the thrust moment of the blade, the lifting moment generated by the lifting force of the blade, the torsional reset moment of the blade, the tilting moment caused by the rotor tilting of the blade, the bending moment caused by the bending of the blade and the unbalanced aerodynamic load.

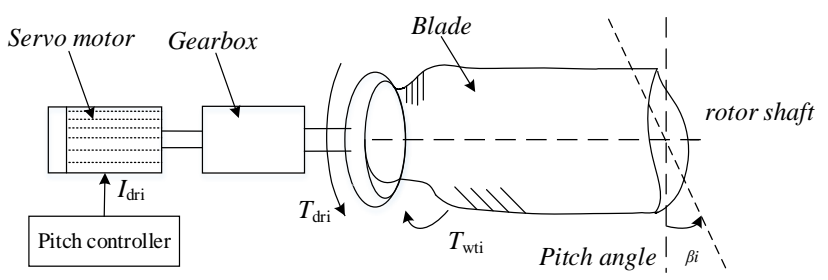

Fig. 1. Pitch mechanism schematic diagram for the ith blade

Derived from the differential form of the momentum moment theorem, for the blade $i$, the dynamic equation of the blade can be obtained as,

$$
\frac{d\left(J_{i} \frac{d \beta_{i}}{d t}\right)}{d t}=T_{d r i}-\frac{d\left(\left(k_{D i}+k_{F i}\right) \beta_{i}\right)}{d t}-T_{t w i}
$$

Equation (1) can be further rewritten as:

$$
\begin{aligned}
J_{i} \ddot{\beta}_{i}+\left(\frac{d J_{i}}{d t}+k_{D i}\right. & \left.+k_{F i}\right) \dot{\beta}_{i} \\
& +\left(\frac{d k_{D i}}{d t}+\frac{d k_{F i}}{d t}\right) \beta_{i}+T_{t w i}=T_{d r i}
\end{aligned}
$$

Where $J_{i}$ is the moment of inertia of the ith blade along its axis; $k_{D i}$ is the damping coefficient of the ith blade; $k_{F i}$ is the friction coefficient of the ith blade. These parameters may change over time during the operation of the blade system.

The relationship between the motor current and the blade driving torque can be written as $T_{d r i}=f\left(I_{d r i}\right)$. For simplicity, a linear relationship is used, $f\left(I_{d r i}\right)=C_{T i} I_{d r i}$, where $C_{T i}$ is an electromechanical transformation constant between the motor driving current of the ith blade and the blade driving torque.

To facilitate the design of the controller, (2) is further rewritten as

$$
\boldsymbol{J}(\boldsymbol{\beta}) \ddot{\beta}+\boldsymbol{M}(\boldsymbol{\beta}, \dot{\boldsymbol{\beta}}) \dot{\boldsymbol{\beta}}+\boldsymbol{Q}(\boldsymbol{\beta}, \dot{\boldsymbol{\beta}}) \boldsymbol{\beta}+\boldsymbol{T}_{t w}(\boldsymbol{\beta}, \dot{\boldsymbol{\beta}})=\boldsymbol{C}_{T} \boldsymbol{u}(3)
$$

Where $\beta=\left[\beta_{1}, \beta_{2}, \beta_{3}\right]^{T} \in \boldsymbol{R}^{3} \quad, \quad \boldsymbol{u}=\left[u_{1}, u_{2}, u_{3}\right]^{T}=$
$\left[I_{d r 1}, I_{d r 2}, I_{d r 3}\right]^{T} \in \boldsymbol{R}^{3}, \boldsymbol{T}_{t w}(\boldsymbol{\beta}, \dot{\boldsymbol{\beta}})=\left[T_{t w 1}, T_{t w 2}, T_{t w 3}\right]^{T} \in$ $\boldsymbol{R}^{3}, \quad \boldsymbol{J}(\boldsymbol{\beta})=\operatorname{diag}\left[J_{1}, J_{2}, J_{3}\right] \in \boldsymbol{R}^{3 \times 3}, \boldsymbol{M}(\boldsymbol{\beta}, \dot{\boldsymbol{\beta}})=$ $\operatorname{diag}\left[M_{1}, M_{2}, M_{3}\right] \in \boldsymbol{R}^{3 \times 3} \quad, \quad \boldsymbol{Q}(\boldsymbol{\beta}, \dot{\boldsymbol{\beta}})=\operatorname{diag}\left[Q_{1}, Q_{2}, Q_{3}\right] \in$ $\boldsymbol{R}^{3 \times 3}$, and $M_{i}=\frac{d J_{i}}{d t}+k_{D i}+k_{F i}, i=1,2,3 ; Q_{i}==\frac{d k_{D i}}{d t}+$ $\frac{d k_{F i}}{d t}, i=1,2,3 \quad ; \quad \boldsymbol{C}_{\boldsymbol{T}}=\operatorname{diag}\left[C_{T 1}, C_{T 2}, C_{T 3}\right] \in \boldsymbol{R}^{3 \times 3} \quad$ is a positive constant diagonal matrix. Define $\boldsymbol{x}_{1}=\boldsymbol{\beta}, \boldsymbol{x}_{2}=\dot{\boldsymbol{\beta}}$, (3) can be reorganized in the following form:

$$
\left\{\begin{array}{c}
\dot{\boldsymbol{x}}_{1}=\boldsymbol{F}_{1}\left(\overline{\boldsymbol{x}}_{1}\right)+\boldsymbol{G}_{1}\left(\overline{\boldsymbol{x}}_{1}\right) \boldsymbol{x}_{2}+\boldsymbol{D}_{1}\left(\overline{\boldsymbol{x}}_{1}, t\right) \\
\dot{\boldsymbol{x}}_{2}=\boldsymbol{F}_{2}\left(\overline{\boldsymbol{x}}_{2}\right)+\boldsymbol{G}_{2}\left(\overline{\boldsymbol{x}}_{2}\right) \boldsymbol{B}(\xi) \boldsymbol{u}+\boldsymbol{D}_{2}\left(\overline{\boldsymbol{x}}_{2}, t\right) \\
\boldsymbol{y}=\boldsymbol{x}_{1} .
\end{array}\right.
$$

Where $\boldsymbol{x}_{i} \in \boldsymbol{R}^{3}, \overline{\boldsymbol{x}}_{i}=\left[\boldsymbol{x}_{1}^{T}, \ldots, \boldsymbol{x}_{i}^{T}\right] \in \boldsymbol{R}^{i \times 3}, i=1,2$ are the states vectors, it is noted that those states are available for the controller design. $\boldsymbol{F}_{i}\left(\overline{\boldsymbol{x}}_{i}\right)$ represents the uncertainties. $\boldsymbol{G}_{2}\left(\overline{\boldsymbol{x}}_{2}\right)=\boldsymbol{J}^{-1}\left(\boldsymbol{x}_{1}\right) \boldsymbol{C}_{\boldsymbol{T}}, \quad \boldsymbol{G}_{1}\left(\boldsymbol{x}_{1}\right)=\boldsymbol{I}_{3 \times 3}$ represents the unknown state-independent control gain matrix. Moreover, $\boldsymbol{D}_{1}\left(\boldsymbol{x}_{1}, t\right)$ and $\boldsymbol{D}_{2}\left(\overline{\boldsymbol{x}}_{2}, t\right)$ represents the unknown external disturbances. $\boldsymbol{u}$ represents the actual control input. $\boldsymbol{B}(\xi)=$ $\operatorname{diag}\left\{B_{i}\left(\xi_{i}\right)\right\}$ is a bounded dialog matrix related to the transformed error $\xi$, which will be discussed later. Besides, there exist some positive constants $B_{i}$ and $\bar{B}_{i}$ such that $0<$ $B_{i} \leq B_{i}\left(\xi_{i}\right) \leq \bar{B}_{i}$.

In order for the system to realize a feasible performance, the following assumptions are established.

Assumption 1: The desired pitch angle signal. is a smooth function of $t$, and $\dot{\boldsymbol{y}}_{\boldsymbol{d}}, \ddot{\boldsymbol{y}}_{\boldsymbol{d}}$ are known and bounded.

Assumption 2: For any given positive constants $E_{1}>0$, it holds that $V(0) \leq E_{1}$, where $V(0)$ is the initial of the Lyapunov function, which is detailed in Section 3.

Assumption 3: For the unknown and uncertain disturbance $\boldsymbol{D}_{i}$, there exists an unknown constant $\bar{d}_{i}$ and a known continuous scalar function $F_{d i}\left(\bar{x}_{i}\right)$ such that $\left\|\boldsymbol{D}_{i}\right\| \leq$ $\bar{d}_{i} F_{d i}\left(\bar{x}_{i}\right)$ for all $t \geq 0$.

Assumption 4: The leading minors $\Delta_{1}, \Delta_{2}, \ldots, \Delta_{m}$ for the $3 \times 3$ control gain matrix $\boldsymbol{G}\left(\overline{\boldsymbol{x}}_{2}\right)$ are nonzero. Besides, the sign of $\boldsymbol{G}\left(\overline{\boldsymbol{x}}_{2}\right)$ is known and available for the controller design.

Assumption 5: The desired signal $\boldsymbol{y}_{\boldsymbol{d}}$ satisfy that $\left\|\boldsymbol{y}_{d}\right\| \leq$ $Y_{0} \leq k_{c 1}$, where $k_{c 1}$ and $Y_{0}$ are some known positive constants[31].

Remark 1: Assumptions 1 and 2 are widely used in dynamic surface control methods[32]. From Assumption 1, there exists a positive constant $E_{0}$ such that $\boldsymbol{\Pi}_{0}=$ $\left\{\left(\boldsymbol{y}_{d}, \dot{\boldsymbol{y}}_{d}, \ddot{\boldsymbol{y}}_{d}\right):\left\|\boldsymbol{y}_{d}\right\|^{2}+\left\|\dot{\boldsymbol{y}}_{d}\right\|^{2}+\left\|\ddot{\boldsymbol{y}}_{d}\right\|^{2} \leq E_{0}\right.$. Assumption 3 denotes that the external disturbance is bounded by an unknown constant $\bar{d}_{i}$ and a known continuous scalar function $F_{d i}\left(\bar{x}_{i}\right)$, where $F_{d i}\left(\bar{x}_{i}\right)$ is bounded only when the system states $\bar{x}_{i}\left(x_{1}, x_{2}, \ldots, x_{i}\right)$ are bounded. Assumption 4 is common in the controller design for a multi-input multioutput (MIMO) system. Assumption 3 on the trajectory is needed to ensure that the output constraints are not violated.

Lemma 1 [29]: for the $3 \times 3$ control gain matrix $\boldsymbol{G}$ with nonzero leading minors $\Delta_{1}, \Delta_{2}, \Delta_{3}$ can be factored as $\boldsymbol{G}=$ $\boldsymbol{S} \boldsymbol{C} \boldsymbol{U}$, where $\boldsymbol{S}$ is symmetric and positive definite, $\boldsymbol{U}$ is unity upper triangular, and $\boldsymbol{C}=\boldsymbol{Q} \boldsymbol{R}$ is diagonal, where $\boldsymbol{R}=$ $\operatorname{diag}\left\{\operatorname{sign}\left(\Delta_{1}\right), \operatorname{sign}\left(\Delta_{2} \Delta_{1}^{-1}\right), \operatorname{sign}\left(\Delta_{3} \Delta_{2}^{-1}\right)\right\}$ and $\boldsymbol{Q}$ being an arbitrary positive diagonal matrix. 


\section{Controller Design}

For the individual pitch controller of WT, the goal is to ensure the pitch angles $\boldsymbol{\beta}=\left[\beta_{1}, \beta_{2}, \beta_{3}\right]^{T}$ of the three blades independently track the desired pitch angles $\boldsymbol{\beta}^{*}=$ $\left[\beta_{1}^{*}, \beta_{2}^{*}, \beta_{3}^{*}\right]^{T}$. To proceed, a tracking error model introduced [30],

$$
\left\{\begin{array}{c}
z_{1}=x_{1}-y_{d} \\
z_{2}=x_{2}-\alpha_{2 f} \\
y_{2}=\alpha_{2 f}-\alpha_{1}
\end{array}\right.
$$

Where $\boldsymbol{\alpha}_{2 f}$ is the output of the first-order filter with $\boldsymbol{\alpha}_{1}$ as the input, and $\boldsymbol{y}_{\boldsymbol{d}}=\boldsymbol{\beta}^{*}$.

Note that from (5), we have:

$$
x_{2}=z_{2}+y_{2}+\alpha_{1}
$$

To make sure that the error vector $\boldsymbol{z}_{1}$ converges within a finite time, we define the following rate function[20]:

$$
\beta(t)=\left\{\begin{array}{c}
\frac{T^{3} \kappa(t)}{\left(1-b_{f}\right)(T-t)^{3}+b_{f} T^{3} \kappa(t)}, 0 \leq t<T \\
\frac{1}{b_{f}}, t \geq T
\end{array}\right.
$$

where $0<b_{f} \ll 1$ is a design parameter; $T>0$ is a finite settling time chosen by the designer; $\kappa(t) \in C^{\infty}$ is a strictly increasing function, which is relevant to the convergence rate of the tracking error.

Remark 2: Note that $\beta(t) \in\left[1,1 / b_{f}\right]$ for all $t \geq 0$. $\beta^{-1}$, $\beta, \dot{\beta}$, and $\ddot{\beta}$ are bounded for $t \geq 0$. Moreover, there exists a positive constant $E_{\beta}$ such that $\boldsymbol{\Pi}_{\beta}=\left\{(\beta, \dot{\beta}, \ddot{\beta}): \beta^{2}+\dot{\beta}^{2}+\right.$ $\left.\ddot{\beta}^{2} \leq E_{\beta}\right\}$.

Integrate (5) with (7) yields:

$$
\left\{\begin{array}{l}
\zeta_{1}=\beta z_{1} \\
\zeta_{2}=\beta z_{2}
\end{array}\right.
$$

where $\boldsymbol{\zeta}_{1}$ and $\boldsymbol{\zeta}_{2}$ are the transformed error used in the controller design to obtain the desired tracking performance.

From (4), the unknown non-linear vector function $\left(\boldsymbol{G}_{1}-\right.$ I) $\boldsymbol{x}_{2}$ is approximated by an RBFNN as follows:

$$
\boldsymbol{F}_{1}+\left(\boldsymbol{G}_{1}-\boldsymbol{I}\right) \boldsymbol{x}_{2}=\boldsymbol{\omega}_{1}^{T} \boldsymbol{\varphi}_{1}\left(\boldsymbol{Z}_{1}\right)+\boldsymbol{\delta}_{1}\left(\boldsymbol{Z}_{1}\right)
$$

Where $\boldsymbol{I} \in \boldsymbol{R}^{3 \times 3}$ is an identity matrix, $\boldsymbol{Z}_{1}=\overline{\boldsymbol{x}}_{2} \in \boldsymbol{R}^{\mathbf{6}}$ is an obtainable stimulating signal vector, $\boldsymbol{\omega}_{1} \in \boldsymbol{R}^{89 \times 6}$ is the ideal weight matrix; $\boldsymbol{\varphi}_{1}\left(\boldsymbol{Z}_{1}\right)$ is the bounded basis function vector; and $\boldsymbol{\delta}_{1}\left(\boldsymbol{Z}_{1}\right)$ denotes the intrinsic approximation error satisfying $\left\|\boldsymbol{\delta}_{1}\left(\boldsymbol{Z}_{1}\right)\right\| \leq \bar{\delta}_{1}<\infty$, with $\bar{\delta}_{1}$ being a small unknown constant.

To further simplify the tedious online computation process, the following virtual parameter is introduced,

$$
\theta=\max \left\{\left\|\omega_{1}\right\|^{2}\right\}
$$

Now, we proceed to design the virtual and actual control laws.

Step 1: The virtual control law will be derived in this step.

Note that $\boldsymbol{z}_{1}=\boldsymbol{x}_{1}-\boldsymbol{y}_{d}$, then we have

$$
\begin{aligned}
\dot{\boldsymbol{z}}_{1} & =\boldsymbol{F}_{1}+\boldsymbol{G}_{1} \boldsymbol{x}_{2}+\boldsymbol{D}_{1}-\dot{\boldsymbol{y}}_{d} \\
& =\boldsymbol{F}_{1}+\left(\boldsymbol{G}_{1}-\boldsymbol{I}\right) \boldsymbol{x}_{2}+\boldsymbol{x}_{2}+\boldsymbol{D}_{1}-\dot{\boldsymbol{y}}_{d}
\end{aligned}
$$

From (9), we have

$$
\dot{\boldsymbol{z}}_{1}=\boldsymbol{\omega}_{1}^{T} \boldsymbol{\varphi}_{1}+\boldsymbol{\delta}_{1}+\boldsymbol{z}_{2}+\boldsymbol{y}_{2}+\boldsymbol{\alpha}_{1}+\boldsymbol{D}_{1}-\dot{\boldsymbol{y}}_{d}
$$

For $i=1$, from (8), we can further get the following transformed dynamics:

$$
\begin{aligned}
\dot{\boldsymbol{\zeta}}_{1} & =\dot{\beta} \mathbf{z}_{1}+\beta \dot{\boldsymbol{z}}_{1} \\
& =\beta\left(\beta^{-1} \dot{\beta} \mathbf{z}_{1}+\boldsymbol{\omega}_{1}^{T} \boldsymbol{\varphi}_{1}+\boldsymbol{\delta}_{1}+\boldsymbol{z}_{2}+\boldsymbol{y}_{2}+\boldsymbol{\alpha}_{1}+\boldsymbol{D}_{1}-\dot{\boldsymbol{y}}_{d}\right) \\
& =\beta\left(\beta^{-1} \dot{\beta} \mathbf{z}_{1}+\boldsymbol{z}_{2}+\boldsymbol{\alpha}_{1}-\dot{\boldsymbol{y}}_{d}\right)+\beta\left(\boldsymbol{\omega}_{1}^{T} \boldsymbol{\varphi}_{1}+\boldsymbol{\delta}_{1}+\boldsymbol{y}_{2}+\boldsymbol{D}_{1}\right)
\end{aligned}
$$

By introducing a new state variable $\boldsymbol{\alpha}_{1}$ and letting $\boldsymbol{\alpha}_{2 f}$ pass through a first-order filter with a positive constant $\tau_{2}$ yields [32],

$$
\tau_{2} \dot{\alpha}_{2 f}+\alpha_{2 f}=\alpha_{1}, \alpha_{2 f}(0)=\alpha_{1}(0)
$$

Where $\boldsymbol{\alpha}_{2 f}(0)$ is the initial value of $\boldsymbol{\alpha}_{2 f}$.

To ensure that the constraint $\boldsymbol{x}_{1}$ is not violated, the following barrier Lyapunov function (BLF) is introduced:

$$
V_{10}=\frac{1}{2} \log \frac{k_{a}^{2}}{k_{a}^{2}-\zeta_{1}^{T} \zeta_{1}}
$$

To meet the restriction of $\left\|x_{1}\right\|<k_{c 1}$, with $k_{c 1}$ being given, $k_{a}$ must be chosen such that

$$
k_{a}=k_{c 1}-Y_{0}
$$

where $Y_{0}$ is defined as in assumption 3. Consider the following Lyapunov function candidate:

$$
V_{1}=V_{10}+\frac{1}{2} \boldsymbol{y}_{2}^{T} \boldsymbol{y}_{2}
$$

Then, the derivative of $V_{1}$ along (17) is

$$
\begin{aligned}
\dot{V}_{1}= & M_{1} \boldsymbol{\zeta}_{1}^{T} \dot{\boldsymbol{\zeta}}_{1}+\boldsymbol{y}_{2}^{T} \dot{\boldsymbol{y}}_{2} \\
= & M_{1} \boldsymbol{\zeta}_{1}^{T} \beta\left(\beta^{-1} \dot{\beta} \mathbf{z}_{1}+\boldsymbol{z}_{2}+\boldsymbol{\alpha}_{1}-\dot{\boldsymbol{y}}_{d}\right) \\
& +M_{1} \boldsymbol{\zeta}_{1}^{T} \beta\left(\boldsymbol{\omega}_{1}^{T} \boldsymbol{\varphi}_{1}+\boldsymbol{\delta}_{1}+\boldsymbol{y}_{2}+\boldsymbol{D}_{1}\right)+\boldsymbol{y}_{2}^{T} \dot{\boldsymbol{y}}_{2}
\end{aligned}
$$

where $M_{1}=\left(1 /\left(k_{a}^{2}-\zeta_{1}^{T} \zeta_{1}\right)\right)$, and using the following inequalities:

$$
\begin{gathered}
M_{1} \zeta_{1}^{T} \beta \boldsymbol{\omega}_{1}^{T} \varphi_{1} \leq \frac{M_{1}^{2} \beta^{2}\left\|\zeta_{1}\right\|^{2}\left\|\boldsymbol{\omega}_{1}\right\|^{2}\left\|\boldsymbol{\varphi}_{1}\right\|^{2}}{2 r_{1}^{2}}+\frac{r_{1}^{2}}{2} \\
\leq \frac{\theta M_{1}^{2} \beta^{2}\left\|\zeta_{1}\right\|^{2}\left\|\boldsymbol{\varphi}_{1}\right\|^{2}}{2 r_{1}^{2}}+\frac{r_{1}^{2}}{2} \\
M_{1} \zeta_{1}^{T} \beta \boldsymbol{\delta}_{1} \leq \frac{M_{1}^{2} \beta^{2}\left\|\zeta_{1}\right\|^{2}}{2}+\frac{\delta_{1}^{2}}{2} \\
M_{1} \zeta_{1}^{T} \beta \boldsymbol{y}_{2} \leq \frac{M_{1}^{2} \beta^{2}\left\|\zeta_{1}\right\|^{2}}{2}+\frac{1}{2}\left\|\boldsymbol{y}_{2}\right\|^{2} \\
M_{1} \zeta_{1}^{T} \beta \boldsymbol{D}_{1} \leq M_{1} \beta\left\|\zeta_{1}\right\|\left\|\boldsymbol{D}_{1}\right\| \leq M_{1} \beta\left\|\zeta_{1}\right\| d_{1} F_{d 1} \\
\leq \frac{M_{1}^{2} \beta^{2}\left\|\zeta_{1}\right\|^{2} F_{d 1}^{2}}{2}+\frac{d_{1}^{2}}{2}
\end{gathered}
$$

where $r_{1}>0$ is the design parameter, $\bar{d}_{1}$ and $F_{d 1}$ are defined in assumption 2

From (18), we have, 


$$
\begin{aligned}
\dot{V}_{1} \leq & M_{1} \zeta_{1}^{T} \beta\left(\beta^{-1} \dot{\beta} z_{1}+z_{2}+\boldsymbol{\alpha}_{1}-\dot{\boldsymbol{y}}_{d}+\frac{\theta M_{1} \beta \boldsymbol{z}_{1}\left\|\boldsymbol{\varphi}_{1}\right\|^{2}}{2 r_{1}^{2}}\right. \\
+M_{1} \beta \zeta_{1}(1+ & \left.\left.\frac{1}{2} F_{d 1}^{2}\right)\right)+\boldsymbol{y}_{2}^{T} \dot{\boldsymbol{y}}_{2}+d \frac{1}{2}\left\|\boldsymbol{y}_{2}\right\|^{2} \\
& +\frac{r_{1}^{2}}{2}+\frac{\bar{\delta}_{1}^{2}}{2}+\frac{\bar{d}_{1}^{2}}{2}
\end{aligned}
$$

Then, a virtual control law $\alpha_{1}$ can be constructed as,

$$
\begin{gathered}
\boldsymbol{\alpha}_{1}=-k_{1} \mathbf{z}_{1}-\beta^{-1} \dot{\beta} \mathbf{z}_{1}+\dot{\boldsymbol{y}}_{d}-\frac{\widehat{\theta} M_{1} \beta \zeta_{1}\left\|\boldsymbol{\varphi}_{1}\right\|^{2}}{2 r_{1}^{2}} \\
-M_{1} \beta \zeta_{1}\left(1+\frac{1}{2} F_{d 1}^{2}\right)
\end{gathered}
$$

where $k_{1}>0$ is the design parameters and $\hat{\theta}$ is the estimation of $\theta$ to be specified later.

By substituting (24) into (23), we obtain

$$
\begin{aligned}
\dot{V}_{1} \leq-k_{1} M_{1} \zeta_{1}^{T} \zeta_{1} & +M_{1} \zeta_{1}^{T} \zeta_{2}+\frac{\widetilde{\theta} M_{1}^{2} \beta^{2}\left\|\zeta_{1}\right\|^{2}\left\|\varphi_{1}\right\|^{2}}{2 r_{1}^{2}} \\
& +\boldsymbol{y}_{2}^{T} \dot{\boldsymbol{y}}_{2}+\frac{1}{2}\left\|\boldsymbol{y}_{2}\right\|^{2}+D_{1}
\end{aligned}
$$

where $\tilde{\theta}=\theta-\widehat{\theta}$ is the estimation error, and $\bar{D}_{1}=$ $\left(r_{1}^{2} / 2\right)+\left(\bar{\delta}_{1}^{2} / 2\right)+\left(\bar{d}_{1}^{2} / 2\right)$.

By using (5) and (14), it is seen that

$$
\dot{\boldsymbol{y}}_{2}=\dot{\boldsymbol{\alpha}}_{2 f}-\dot{\boldsymbol{\alpha}}_{1}=-\frac{\boldsymbol{y}_{2}}{\tau_{2}}-\dot{\boldsymbol{\alpha}}_{1}
$$

Note that $\dot{\boldsymbol{\alpha}}_{1} \boldsymbol{\varepsilon}(\boldsymbol{u})\left\|\dot{\boldsymbol{\alpha}}_{1}\right\| \leq a_{1}\left(\boldsymbol{x}_{1}, \boldsymbol{x}_{2}, \beta, \dot{\beta}, \hat{\theta}, \boldsymbol{y}_{d}, \dot{\boldsymbol{y}}_{d}\right)$ is the function of $\boldsymbol{x}_{1}, \boldsymbol{x}_{2}, \beta, \dot{\beta}, \hat{\theta}, \boldsymbol{y}_{d}, \dot{\boldsymbol{y}}_{d}$, and there exists a continuous function $a_{1}\left(\boldsymbol{x}_{1}, \boldsymbol{x}_{2}, \beta, \dot{\beta}, \hat{\theta}, \boldsymbol{y}_{d}, \dot{\boldsymbol{y}}_{d}\right)$, such that

$$
\left\|\dot{\boldsymbol{\alpha}}_{1}\right\| \leq a_{1}\left(\boldsymbol{x}_{1}, \boldsymbol{x}_{2}, \beta, \dot{\beta}, \hat{\theta}, \boldsymbol{y}_{d}, \dot{\boldsymbol{y}}_{d}\right)
$$
have

Hence, from (26), and by using Young's inequality, we

$$
\boldsymbol{y}_{2}^{T} \dot{\boldsymbol{y}}_{2}=\boldsymbol{y}_{2}^{T}\left(-\frac{y_{2}}{\tau_{2}}-\dot{\alpha}_{1}\right) \leq-\frac{1}{\tau_{2}}\left\|\boldsymbol{y}_{2}\right\|^{2}+\frac{a_{1}^{2}}{2}
$$

Thus, from (25), we get

$$
\begin{gathered}
\dot{V}_{1} \leq-k_{1} M_{1} \zeta_{1}^{T} \zeta_{1}+M_{1} \zeta_{1}^{T} \zeta_{2}+\frac{\widetilde{\theta} M_{1}^{2} \beta^{2}\left\|\zeta_{1}\right\|^{2}\left\|\varphi_{1}\right\|^{2}}{2 r_{1}^{2}} \\
-\frac{1}{\tau_{2}}\left\|\boldsymbol{y}_{2}\right\|^{2}+\left\|\boldsymbol{y}_{2}\right\|^{2}+\frac{a_{1}^{2}}{2}+D_{1} \\
\leq-k_{1} M_{1} \zeta_{1}^{T} \zeta_{1}+M_{1} \zeta_{1}^{T} \zeta_{2}+\frac{\widetilde{\theta} M_{1}^{2} \beta^{2}\left\|\zeta_{1}\right\|^{2}\left\|\varphi_{1}\right\|^{2}}{2 r_{1}^{2}} \\
-\tau_{2}\left\|\boldsymbol{y}_{2}\right\|^{2}+\frac{a_{1}^{2}}{2}+D_{1}
\end{gathered}
$$

where the design parameter $\tau_{2}$ satisfies that $\frac{1}{\tau_{2}} \geq 1+\tau_{2}^{*}$ , with $\tau_{2}^{*}$ a positive constant.

Step 2: The final control law $\boldsymbol{u}$ will be derived in this step. Note that $\boldsymbol{z}_{2}=\boldsymbol{x}_{2}-\boldsymbol{\alpha}_{2 f}$ we have

$$
\begin{aligned}
\dot{\boldsymbol{z}}_{2} & =\dot{\boldsymbol{x}}_{2}-\dot{\boldsymbol{\alpha}}_{2 f} \\
& =\boldsymbol{F}_{2}+\boldsymbol{G}_{2} \boldsymbol{B} \boldsymbol{u}+\boldsymbol{G}_{2} \epsilon+\boldsymbol{D}_{2}-\dot{\boldsymbol{\alpha}}_{2 f} .
\end{aligned}
$$

From (8), we have,

$$
\begin{aligned}
& \dot{\boldsymbol{\zeta}}_{2}=\dot{\beta} \boldsymbol{z}_{2}+\beta \dot{\boldsymbol{z}}_{2} \\
& =\beta \boldsymbol{G}_{2} \boldsymbol{B} \boldsymbol{u}+\beta\left(\beta^{-1} \dot{\beta} \boldsymbol{z}_{2}+\boldsymbol{F}_{2}+\boldsymbol{G}_{2} \epsilon+\boldsymbol{D}_{2}-\dot{\boldsymbol{\alpha}}_{2 f}\right)
\end{aligned}
$$

Where $\boldsymbol{G}_{2}\left(\bar{x}_{2}\right)$ can be decomposed as[33],

$$
\boldsymbol{G}_{2}\left(\bar{x}_{2}\right)=\boldsymbol{S}\left(\bar{x}_{2}\right) \boldsymbol{C} \boldsymbol{U}\left(\bar{x}_{2}\right)
$$

where the matrix $\boldsymbol{S}\left(\bar{x}_{2}\right)$ is symmetric and positive definite, $\boldsymbol{U}\left(\bar{x}_{2}\right)$ is unity upper triangular, and $\boldsymbol{C}=\boldsymbol{Q} \boldsymbol{R}$ is diagonal, where $\boldsymbol{R}=\operatorname{diag}\left\{\operatorname{sign}\left(\Delta_{1}\right), \operatorname{sign}\left(\Delta_{2} \Delta_{1}^{-1}\right), \operatorname{sign}(\right.$ $\left.\left.\Delta_{3} \Delta_{2}^{-1}\right)\right\}$ and $\boldsymbol{Q}$ being an arbitrary positive diagonal matrix.

Then, we choose

$$
\boldsymbol{Q}=\operatorname{diag}\left\{\frac{1}{\underline{B}_{1}}, \ldots, \frac{1}{\underline{B}_{3}}\right\}
$$

The elements of $\boldsymbol{C}$ denoted by $C_{i}$ satisfying [34]

$$
\left|C_{i}\right| B_{i} \geq 1,(i=1,2,3)
$$

Since the sign of $\Delta_{i}$ is known, $\operatorname{sign}\left(C_{i}\right)$ is known. Then, from (31), we have

$$
\begin{aligned}
\boldsymbol{S}^{-1} \dot{\boldsymbol{\zeta}}_{2}= & \beta \boldsymbol{C} \boldsymbol{U} \boldsymbol{B} \boldsymbol{u}+\boldsymbol{S}^{-1} \beta\left(\beta^{-1} \dot{\beta} \mathbf{z}_{2}+\boldsymbol{F}_{2}+\boldsymbol{G}_{2} \epsilon+\boldsymbol{D}_{2}-\dot{\boldsymbol{\alpha}}_{2 f}\right) \\
=\beta \boldsymbol{C} \boldsymbol{B} \boldsymbol{u} & +\beta \boldsymbol{C}(\boldsymbol{U}-\boldsymbol{I}) \boldsymbol{B} \boldsymbol{u}+\boldsymbol{S}^{-1} \beta \\
& \times\left(\beta^{-1} \dot{\beta} \mathbf{z}_{2}+\boldsymbol{F}_{2}+\boldsymbol{G}_{2} \epsilon+\boldsymbol{D}_{2}-\dot{\boldsymbol{\alpha}}_{2 f}\right)
\end{aligned}
$$

Note that $\boldsymbol{U}-\boldsymbol{I}$ is strictly upper triangular and $\boldsymbol{B}$ is a positive diagonal matrix, and we get

$$
\beta \boldsymbol{C}(\boldsymbol{U}-\boldsymbol{I}) \boldsymbol{B} \boldsymbol{u}=\left[\boldsymbol{\Lambda}_{1}^{T} \boldsymbol{\gamma}_{1}, \boldsymbol{\Lambda}_{2}^{T} \boldsymbol{\gamma}_{2}, 0\right]^{T}
$$

where $\boldsymbol{\Lambda}_{1}^{T}=\beta C_{1}\left[U_{1,2} B_{2}, U_{1,3} B_{3}\right], \boldsymbol{\Lambda}_{2}^{T}=\beta C_{2} U_{2,3} B_{3}$, with $U_{i, j}$ being the element of the matrix $\boldsymbol{U}$, and $\boldsymbol{\gamma}_{1}=\left[u_{2}, u_{3}\right]^{T} \in$ $\boldsymbol{R}^{2}, \gamma_{2}=\left[u_{3}\right]^{T} \in \boldsymbol{R}$. For convenience, let $\boldsymbol{\Lambda}_{3}=0$ and $\boldsymbol{\gamma}_{3}=$ 0 . Consider the Lyapunov function candidate

$$
V_{2}=\frac{1}{2} \boldsymbol{\zeta}_{2}^{T} \boldsymbol{S}^{-1} \boldsymbol{\zeta}_{2}
$$

Integrated (37) with (35) and (36), the time derivative of $V_{2}$ is given by

$$
\begin{aligned}
\left(\beta^{-1} \dot{\beta} \mathbf{z}_{2}+\boldsymbol{F}_{2}+\boldsymbol{G}_{2} \epsilon+\boldsymbol{D}_{2}-\dot{\boldsymbol{\alpha}}_{2 f}\right)+\frac{1}{2} \boldsymbol{\zeta}_{2}^{T} \dot{\boldsymbol{S}}^{-1} \boldsymbol{\zeta}_{2} \\
=-k_{2} \boldsymbol{\zeta}_{2}^{T} S^{-1} \boldsymbol{\zeta}_{2}+\beta \boldsymbol{\zeta}_{2}^{T} \boldsymbol{C} \boldsymbol{B} \boldsymbol{u}+\sum_{i=1}^{3} \zeta_{3 i} \boldsymbol{\Lambda}_{i}^{T} \boldsymbol{\gamma}_{i}+\frac{1}{2} \boldsymbol{\zeta}_{2}^{T} \dot{\boldsymbol{S}}^{-1} \boldsymbol{\zeta}_{2} \\
+\boldsymbol{\zeta}_{2}^{T} \boldsymbol{S}^{-1} \beta\left(k_{2} \mathbf{z}_{2}+\beta^{-1} \dot{\beta} \mathbf{z}_{2}-\dot{\boldsymbol{\alpha}}_{2 f}+\boldsymbol{F}_{2}+\boldsymbol{G}_{2} \epsilon+\boldsymbol{D}_{2}\right)
\end{aligned}
$$

where $k_{2}>0$ is a user-defined parameter. We denote the ith rows of $\boldsymbol{S}^{-1}$ and $\dot{\boldsymbol{S}}^{-1}$ as $\boldsymbol{\eta}_{i}$ and $\overline{\boldsymbol{\eta}}_{i}$, respectively. Noting that $\boldsymbol{B}_{i}$, and disturbance $\boldsymbol{D}_{2}$ are bounded, and it is readily obtained that there exist continuous but unknown functions $L_{i}\left(\bar{x}_{2}\right) \in R, i=1,2,3$ such that $\max \left\{\left\|\boldsymbol{\Lambda}_{i}\right\|,\left\|\boldsymbol{\eta}_{i}\right\|,\left\|\boldsymbol{\eta}_{i}\right\|\left\|\boldsymbol{F}_{2}\right\|\right.$, $\left.\left\|\boldsymbol{\eta}_{i}\right\|\left\|\overline{\boldsymbol{G}}_{2}\right\| \bar{\epsilon}, d_{2}\left\|\boldsymbol{\eta}_{i}\right\|,\left\|\overline{\boldsymbol{\eta}}_{i}\right\|\right\} \leq L_{i}\left(\bar{x}_{2}\right)$. Then, from (38), we further have

$$
\begin{gathered}
\dot{V}_{2}=-k_{2} \boldsymbol{\zeta}_{2}^{T} S^{-1} \boldsymbol{\zeta}_{2}+\beta \boldsymbol{\zeta}_{2}^{T} \boldsymbol{C B} \boldsymbol{u}+\sum_{i=1}^{3} \zeta_{2 i} \\
\times\left(\boldsymbol{\Lambda}_{i}^{T} \boldsymbol{\gamma}_{i}+\eta_{i} \beta \times\left(k_{2} \boldsymbol{z}_{2}+\beta^{-1} \dot{\beta} \mathbf{z}_{2}\right.\right. \\
\left.\left.-\dot{\boldsymbol{\alpha}}_{2 f}+\boldsymbol{F}_{2}+\boldsymbol{G}_{2} \boldsymbol{\varepsilon}+\boldsymbol{D}_{2}\right)+\frac{1}{2} \boldsymbol{\eta}_{i} \boldsymbol{\zeta}_{2}\right) \\
\leq-k_{2} \boldsymbol{\zeta}_{2}^{T} \boldsymbol{S}^{-1} \boldsymbol{\zeta}_{2}+\beta \boldsymbol{\zeta}_{2}^{T} \boldsymbol{C B} \boldsymbol{u}+\sum_{i=1}^{3}\left|\zeta_{2 i}\right| \\
\times\left(\left\|\boldsymbol{\Lambda}_{i}\right\|\left\|\boldsymbol{\gamma}_{i}\right\|+\left\|\boldsymbol{\eta}_{i}\right\| \beta \times\left(\left\|k_{2} \boldsymbol{z}_{2}+\beta^{-1} \dot{\beta} \mathbf{z}_{2}-\dot{\boldsymbol{\alpha}}_{2 f}\right\|\right.\right. \\
\left.\left.+\left\|\boldsymbol{F}_{2}\right\|+\left\|\boldsymbol{G}_{2}\right\| \bar{\epsilon}+\bar{d}_{2} F_{d 2}\right)+\frac{1}{2}\left\|\overline{\boldsymbol{\eta}}_{i}\right\|\left\|\boldsymbol{\zeta}_{2}\right\|\right) \\
\leq-k_{2} \boldsymbol{\zeta}_{2}^{T} \boldsymbol{S}^{-1} \boldsymbol{\zeta}_{2}+\beta \boldsymbol{\zeta}_{2}^{T} \boldsymbol{C B u}+\sum_{i=1}^{3} L_{i} \psi_{i}\left|\zeta_{2 i}\right|
\end{gathered}
$$


where

$$
\begin{array}{r}
\psi_{i}=\left\|\boldsymbol{\gamma}_{i}\right\|+\beta\left\|k_{2} \mathbf{z}_{2}+\beta^{-1} \dot{\beta} \mathbf{z}_{2}-\dot{\boldsymbol{\alpha}}_{2 f}\right\| \\
+2 \beta+\beta F_{d 2}+\frac{1}{2}\left\|\zeta_{2}\right\|
\end{array}
$$

$L_{i}\left(\bar{x}_{2}\right)(i=1,2,3)$ is an unknown non-linear continuous function, which can be approximated by an RBFNN with

$$
L_{i}\left(\bar{x}_{2}\right)=\boldsymbol{N}_{i}^{T} \boldsymbol{P}_{i}\left(\bar{x}_{2}\right)+\varepsilon_{i}\left(\bar{x}_{2}\right)
$$

where $\boldsymbol{N}_{i} \in \boldsymbol{R}^{89}$ is an ideal weight vector, $\boldsymbol{P}_{i}\left(\bar{x}_{2}\right)$ is the basis function vector of $\mathrm{NN}$, and $\varepsilon_{i}\left(\bar{x}_{2}\right)$ is the intrinsic approximation error and satisfies $\left|\varepsilon_{i}\left(\bar{x}_{2}\right)\right| \leq \bar{\varepsilon}_{i}<\infty(i=$ $1,2,3)$, with $\bar{\varepsilon}_{i}$ being a small unknown constant.

Substituting (41) into (39), we have

$$
\begin{aligned}
\dot{V}_{2} \leq & -k_{2} \boldsymbol{\zeta}_{2}^{T} \boldsymbol{S}^{-1} \boldsymbol{\zeta}_{2}+\beta \boldsymbol{\zeta}_{2}^{T} \boldsymbol{C B}+ \\
& \sum_{i=1}^{3}\left(\mu_{i}\left|\zeta_{2 i}\right|-\widetilde{\boldsymbol{N}}_{i}^{T} \boldsymbol{P}_{i} \psi_{i}\left|\zeta_{2 i}\right|+\varepsilon_{i} \psi_{i}\left|\zeta_{2 i}\right|\right)
\end{aligned}
$$

where $\mu_{i}=\widehat{\boldsymbol{N}}_{i}^{T} \boldsymbol{P}_{i} \psi_{i}$, and $\widetilde{\boldsymbol{N}}_{i}=\widehat{\boldsymbol{N}}_{i}-\boldsymbol{N}_{i}$ is the estimation error.

Thus, the actual control law is designed as:

$$
u_{i}=-\left(\frac{M_{1}}{2}+\frac{i_{i}}{2} \mu_{i}^{2}+\frac{c_{i}}{2} \psi_{i}^{2}\right) \operatorname{sign}\left(C_{i}\right) \zeta_{2 i}
$$

where $i=1,2,3, \iota_{i}>0$ and $c_{i}>0$ are the design parameters, and $\widehat{\boldsymbol{N}}_{i}, \hat{\theta}$ are updated by the following formula:

$$
\begin{aligned}
& \dot{\hat{\boldsymbol{N}}}_{i}=\boldsymbol{\Gamma}_{i} \boldsymbol{P}_{i} \psi_{i}\left|\zeta_{n i}\right|-\sigma_{i} \boldsymbol{\Gamma}_{i} \widehat{\boldsymbol{N}}_{i} \\
& \dot{\hat{\theta}}=\rho \frac{M_{j}^{2} \beta^{2}\left\|\zeta_{1}\right\|^{2}\left\|\boldsymbol{\varphi}_{1}\right\|^{2}}{2 r_{1}^{2}}-\sigma_{\theta} \hat{\theta}
\end{aligned}
$$

where $\boldsymbol{\Gamma}_{i}=\boldsymbol{\Gamma}_{i}^{T}, \sigma_{i}>0, \rho>0$ and $\sigma_{\theta}>0$ are userdefined design parameters.

The structure of the proposed controller is illustrated in Fig.2. Wherein the function 1 comprise (9), (10), (16) and (24), function 2 comprise (40),(43)-(45). The principle of RBFNN is to approximate $L_{i}\left(\bar{x}_{2}\right)(i=1,2,3)$ and $\boldsymbol{F}_{1}+$ $\left(\boldsymbol{G}_{1}-\boldsymbol{I}\right) \boldsymbol{x}_{2}$. Under the control input $\boldsymbol{u}$, the pitch system can realize a favorable tracking performance.

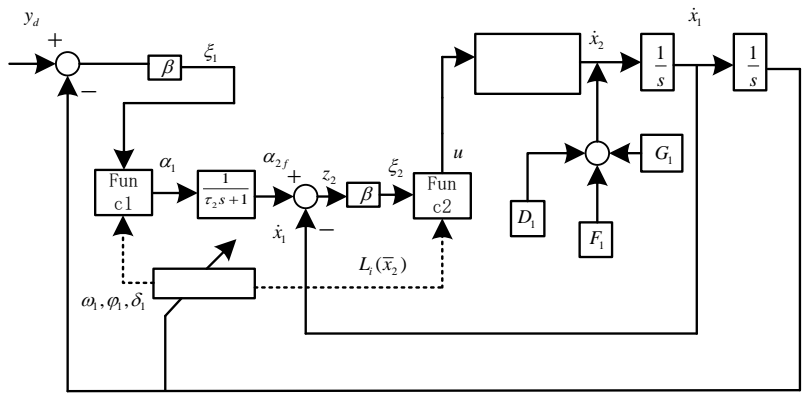

Fig. 2. The diagram of the proposed control method.

The following control objectives are achieved:

1) All the internal signals in the closed-loop system are bounded; the output constraints are not violated, i.e. $\left\|\boldsymbol{x}_{1}\right\|<k_{c 1}, \forall t \geq 0$.

2) The given tracking performance is achieved, such that the tracking error converges to a prescribed compact set around zero within preassigned finite time $T$ at a preset converging rate when $t \in[0, T)$.
Proof: Construct the Lyapunov function candidate as

$$
V=V_{1}+V_{2}+\frac{1}{2 \rho} \tilde{\theta}^{2}+\frac{1}{2} \sum_{i=1}^{3} \widetilde{\boldsymbol{N}}_{i}^{T} \boldsymbol{\Gamma}_{i}^{-1} \widetilde{\boldsymbol{N}}_{i}
$$

Integrating (29) with (42), the time derivative of $V$ is given by

$$
\begin{aligned}
\dot{V} \leq & -k_{1} M_{1} \zeta_{1}^{T} \zeta_{1}+M_{1} \zeta_{1}^{T} \zeta_{2}+\frac{\widehat{\theta} M_{1}^{2} \beta^{2}\left\|\zeta_{1}\right\|^{2}\left\|\boldsymbol{\varphi}_{1}\right\|^{2}}{2 r_{1}^{2}} \\
+ & \tau_{2}^{*}\left\|\boldsymbol{y}_{2}\right\|^{2}+\frac{a_{1}^{2}}{2}+\bar{D}_{1}-k_{2} \zeta_{2}^{T} S^{-1} \zeta_{2} \\
& +\sum_{i=1}^{3}\left(\mu_{i}\left|\zeta_{2 i}\right|-\widetilde{\boldsymbol{N}}_{i}^{T} \boldsymbol{P}_{i} \psi_{i}\left|\zeta_{2 i}\right|+\beta \zeta_{2}^{T} \boldsymbol{C} \boldsymbol{B} \boldsymbol{u}\right. \\
& \left.+\varepsilon_{i} \psi_{i}\left|\zeta_{2 i}\right|\right)-\frac{1}{\rho} \tilde{\theta} \dot{\hat{\theta}}+\sum_{i=1}^{3} \widetilde{\boldsymbol{N}}_{i}^{T} \boldsymbol{\Gamma}_{i}^{-1} \hat{\boldsymbol{N}}_{i} \\
\leq & -k_{1} M_{1} \zeta_{1}^{T} \zeta_{1}+M_{1} \zeta_{1}^{T} \zeta_{2}-k_{2} \zeta_{2}^{T} S^{-1} \zeta_{2} \\
& +\frac{1}{\rho}\left(\rho \tilde{\theta} \frac{\tilde{\theta}_{1}^{2} \beta^{2}\left\|\zeta_{1}\right\|^{2}\left\|\boldsymbol{\varphi}_{1}\right\|^{2}}{2 r_{1}^{2}}-\hat{\hat{\theta}}\right) \\
& +\sum_{i=1}^{3} \beta \zeta_{2 i} C_{i} B_{i} u_{i}+\sum_{i=1}^{3}\left(\mu_{i}\left|\zeta_{2 i}\right|\right. \\
& +\widetilde{\boldsymbol{N}}_{i}^{T} \boldsymbol{\Gamma}_{i}^{-1}\left(\hat{\boldsymbol{N}}_{i}-\boldsymbol{\Gamma}_{i} \boldsymbol{P}_{i} \psi_{i}\left|\zeta_{2 i}\right|+\varepsilon_{i} \psi_{i}\left|\zeta_{2 i}\right|\right) \\
& -\tau_{2}^{*}\left\|\boldsymbol{y}_{2}\right\|^{2}+\frac{a_{1}^{2}}{2}+\bar{D}_{1}
\end{aligned}
$$

Using the following inequalities:

$$
\begin{gathered}
M_{1} \zeta_{1}^{T} \leq \frac{M_{1}}{2} \zeta_{1}^{T} \zeta_{1}+\frac{M_{1}}{2} \zeta_{2}^{T} \zeta_{2} \\
\mu_{i}\left|\zeta_{2 i}\right| \leq \frac{\iota_{i}}{2} \mu_{i}^{2} \zeta_{2 i}^{2}+\frac{1}{2 \iota_{i}} \\
\varepsilon_{i} \psi_{i}\left|\zeta_{2 i}\right| \leq \frac{c_{i}}{2} \psi_{i}^{2} \zeta_{2 i}^{2}+\frac{\varepsilon_{i}^{2}}{2 c_{i}} \leq \frac{c_{i}}{2} \psi_{i}^{2} \zeta_{2 i}^{2}+\frac{\bar{\varepsilon}_{i}^{2}}{2 c_{i}}
\end{gathered}
$$

Substituting (44) and (45) into (47), we then have

$$
\begin{gathered}
\dot{V} \leq-k_{1} M_{1} \zeta_{1}^{T} \zeta_{1}+\frac{M_{1}}{2} \zeta_{1}^{T} \zeta_{1}-k_{2} \zeta_{2}^{T} S^{-1} \zeta_{2} \\
+\frac{\sigma_{\theta}}{\rho} \tilde{\theta} \hat{\theta}+\sum_{i=1}^{3}\left(\frac{1}{2 \iota_{i}}-\sigma_{i} \widetilde{\boldsymbol{N}}_{i}^{T} \widehat{\boldsymbol{N}}_{i}+\frac{\bar{\varepsilon}_{i}^{2}}{2 c_{i}}\right) \\
-\tau_{2}^{*}\left\|\boldsymbol{y}_{2}\right\|^{2}+\frac{a_{1}^{2}}{2}+\bar{D}_{1} .
\end{gathered}
$$

From (44), it is deduced that,

$$
\beta \zeta_{2 i} C_{i} B_{i} u_{i}=-\beta\left|C_{i}\right| B_{i}\left(\frac{M_{1}}{2} \zeta_{2 i}^{2}+\frac{\iota_{i}}{2} \mu_{i}^{2} \zeta_{2 i}^{2}+\frac{c_{i}}{2} \psi_{i}^{2} \zeta_{2 i}^{2}\right)
$$
obtain:

From (35) and $\beta \geq 1$, we have $\beta\left|C_{i}\right| B_{i} \geq 1$, then we

$$
\beta \zeta_{2 i} C_{i} B_{i} u_{i} \leq-\left(\frac{M_{1}}{2} \zeta_{2 i}^{2}+\frac{\iota_{i}}{2} \mu_{i}^{2} \zeta_{2 i}^{2}+\frac{c_{i}}{2} \psi_{i}^{2} \zeta_{2 i}^{2}\right)
$$

Substituting it into (52), we have

$$
\begin{aligned}
\dot{V} \leq & -k_{1} M_{1} \zeta_{1}^{T} \zeta_{1}+\frac{M_{1}}{2} \zeta_{1}^{T} \zeta_{1}-k_{2} \zeta_{2}^{T} S^{-1} \zeta_{2} \\
& +\frac{\sigma_{\theta}}{\rho} \tilde{\theta} \hat{\theta}+\sum_{i=1}^{3}\left(\frac{1}{2 \iota_{i}}-\sigma_{i} \widetilde{\boldsymbol{N}}_{i}^{T} \widehat{\boldsymbol{N}}_{i}+\frac{\bar{\varepsilon}_{i}^{2}}{2 c_{i}}\right) \\
& -\tau_{2}^{*}\left\|\boldsymbol{y}_{2}\right\|^{2}+\frac{a_{j}^{2}}{2}+\bar{D}_{j}
\end{aligned}
$$

Using Young's inequality, we have:

$$
\tilde{\theta} \hat{\theta} \leq-\frac{1}{2} \tilde{\theta}^{2}+\frac{1}{2} \theta^{2}
$$




$$
-\widetilde{\boldsymbol{N}}_{i}^{T} \widehat{\boldsymbol{N}}_{i} \leq-\frac{1}{2} \widetilde{\boldsymbol{N}}_{i}^{T} \widehat{\boldsymbol{N}}_{i}+\frac{1}{2}\left\|\boldsymbol{N}_{i}\right\|^{2}
$$

Note that $x_{1}=z_{1}+y_{d}$ and $x_{2}=z_{2}+y_{2}+\alpha_{1}$, we define a compact set as

$$
\boldsymbol{\Pi}_{1}=\left\{\boldsymbol{\zeta}_{1}, \boldsymbol{\zeta}_{2}, \boldsymbol{y}_{2}, \hat{\theta}, \widehat{\boldsymbol{N}}_{1}, \widehat{\boldsymbol{N}}_{2}, \widehat{\boldsymbol{N}}_{3}: V \leq E_{1}\right\}
$$

As $\boldsymbol{\Pi}_{0} \times \boldsymbol{\Pi}_{1} \times \boldsymbol{\Pi}_{\beta}$ is a compact set in $\boldsymbol{R}^{2 \times(3+1) \times 3+3 \times 89+4}$, it is readily concluded that the continuous function $a_{i}$ has a maximum $\bar{a}_{i}$ on $\boldsymbol{\Pi}_{0} \times \boldsymbol{\Pi}_{1} \times \boldsymbol{\Pi}_{\beta}$, with $\bar{a}_{i}$ being some unknown positive constant.

If $V=C_{1}$ then $a_{i} \leq \bar{a}_{i}$, and substituting (56) and (57) into (55), we can further have

$$
\begin{aligned}
& \dot{V}_{2} \leq-k_{1} M_{1} \zeta_{1}^{T} \zeta_{1}+\frac{M_{1}}{2} \zeta_{1}^{T} \zeta_{1}-k_{2} \zeta_{2}^{T} \boldsymbol{S}^{-1} \zeta_{2}-\frac{\sigma_{\theta}}{2 \rho} \tilde{\theta}^{2} \\
& +\sum_{i=1}^{3}\left(\frac{1}{2 \iota_{i}}-\frac{\sigma_{i}}{2} \widetilde{\boldsymbol{N}}_{i}^{T} \widetilde{\boldsymbol{N}}_{i}+\frac{\sigma_{i}}{2}\left\|\boldsymbol{N}_{i}\right\|^{2}+\frac{\bar{\varepsilon}_{i}^{2}}{2 c_{i}}\right) \\
& +\frac{\sigma_{\theta}}{2 \rho} \theta^{2}-\tau_{2}^{*}\left\|\boldsymbol{y}_{2}\right\|^{2}+\frac{\bar{a}_{1}^{2}}{2}+\bar{D}_{1} \\
& \leq-\left(k_{1}-\frac{1}{2}\right) M_{1} \zeta_{1}^{T} \zeta_{1}-\tau_{2}^{*}\left\|\boldsymbol{y}_{2}\right\|^{2}-k_{2} \zeta_{2}^{T} S^{-1} \zeta_{2} \\
& -\frac{\sigma_{\theta}}{2 \rho} \tilde{\theta}^{2}-\frac{1}{2} \sum_{i=1}^{3} \sigma_{i} \widetilde{\boldsymbol{N}}_{i}^{T} \widetilde{\boldsymbol{N}}_{i}+\frac{\sigma_{\theta}}{2 \rho} \theta^{2} \\
& +\sum_{i=1}^{3}\left(\frac{1}{2 \iota_{i}}+\frac{\sigma_{i}}{2}\left\|\boldsymbol{N}_{i}\right\|^{2}+\frac{\bar{\varepsilon}_{i}^{2}}{2 c_{i}}\right)+\frac{\bar{a}_{1}^{2}}{2}+\bar{D}_{1}
\end{aligned}
$$

Note that

Thus, from (58), we get

$$
-k_{1} M_{1} \zeta_{1}^{T} \zeta_{1} \leq-k_{1} \log \frac{k_{a}^{2}}{k_{a}^{2}-\zeta_{1}^{T} \zeta_{1}}
$$

$$
\dot{V} \leq-\lambda V+\Theta
$$

Where $\lambda=\min \left\{2 k_{q}, 2\left(k_{1}-(1 / 2), 2 \tau_{j}^{*}, \sigma_{\theta}, \sigma_{i} T_{\text {imax }}\right\}\right.$ ( $q$ $=1, j=2, i=1,2,3)$, with $T_{\text {imax }}$ being the largest eigenvalue of the matrix $\boldsymbol{\Gamma}_{i}$ and

$$
\begin{aligned}
\Theta=\left(\frac{\sigma_{\theta}}{2 \rho}\right) \theta^{2} & +\sum_{i=1}^{3}\left(\left(1 / 2 \iota_{i}\right)+\left(\sigma_{i} / 2\right)\left\|\boldsymbol{N}_{i}\right\|^{2}\right. \\
& \left.+\left(\bar{\varepsilon}_{i}^{2} / 2 c_{i}\right)\right)+\bar{a}_{1}^{2} / 2+\bar{D}_{1}<\infty
\end{aligned}
$$

Besides, we can choose $k_{1}>(1 / 2)$ and $\lambda=\Theta / C_{1}$, and based on that, if $V=C_{1}$, then $V(0)=C_{1}, V(0)=C_{1}, \dot{V} \leq$ $0, \forall t \geq 0$. Furthermore, we get

$$
V \leq \frac{\Theta}{\lambda}+\left(V(0)-\frac{\Theta}{\lambda}\right) e^{-\lambda t}
$$

Therefore, we can conclude that $V(t) \in \ell_{\infty}$ then it follows $\left\|\zeta_{1}(0)\right\|<k_{a}$ that $\zeta_{1}$ always remain in the set as long as the initial value $\left\|\boldsymbol{\zeta}_{1}(t)\right\|<k_{a} ; \boldsymbol{\zeta}_{2} \in \ell_{\infty}, \boldsymbol{y}_{2} \in$ $\ell_{\infty}, \widehat{\boldsymbol{N}}_{i} \in \ell_{\infty}, i=1,2,3$, and $\hat{\theta} \in \ell_{\infty}$. As $\beta$ is bounded, then $\zeta_{i} \in \ell_{\infty}(i=1,2)$ imply that $F_{d 1} \in \ell_{\infty}$. Since $\boldsymbol{z}_{1}=\boldsymbol{x}_{1}-\boldsymbol{y}_{d}$ and $\boldsymbol{y}_{d}$ are bounded, so is $\boldsymbol{x}_{1}$, which further implies that $F_{d 1} \in \ell_{\infty}$, since $\beta^{-1}$ and $\dot{\beta}$ are bounded as mentioned before, we get $\alpha_{1} \in \ell_{\infty}$. Since $\boldsymbol{x}_{2}=z_{2}+\boldsymbol{y}_{2}+\boldsymbol{\alpha}_{1}, \boldsymbol{x}_{2}$ is bounded, $F_{d 2}$ is also bounded, which further implies that $\boldsymbol{\alpha}_{2}$ is bounded. Then, in the same way, we can prove that $\dot{\hat{\theta}}, \dot{\hat{N}}_{i}(i=1,2,3)$, and the actual control inputs $\boldsymbol{u}_{i}(i=1,2,3)$ are bounded. Therefore, all the signals in the closed-loop system are bounded.

\section{Simulation results}

Simulations are conducted in Matlab/Simulink in this section to verify the effectiveness of the proposed method. The references of the pitch angles, which are determined by the pitch regulation system to ensure the WT can achieve constant power operation and mitigated dynamic loads, are as shown in Fig.3. (Refer to [15] for more information on the generation of the pitch angle references). Suppose that the reference pitch angle would vary in the simulation, which is consistent with the actual application. The proposed IPC controller must make sure that the real pitch angles can track the references within a predefined finite time under timevarying unknown system parameters and unknown disturbances.

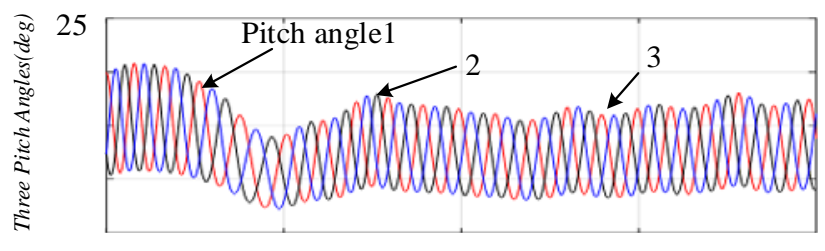

Time $(s)$

Fig. 3. Pitch angle of three blades

In the simulation, the uncertainties of the parameters in the pitch system are simulated by the equations provided in (63). Besides, a sinusoidal signal is used to simulate the aerodynamic load caused by wind shear effects and tower shadow effects as these are periodical. Meanwhile, white noise is used to simulate the random disturbance, as shown in (64). In the simulation, the initial values of the desired trajectory are $x_{1}(0)=x_{2}(0)=[0,0,0]^{T}$, and $\hat{\theta}(0)=0$; the RBFNN used in the controller contains 89 nodes with centers evenly distributed in the span of input space $[-11,11]$. The parameters of the RBFNN controller are listed in Table 1.

Table 1 Simulation parameters

\begin{tabular}{cc}
\hline Parameters & Value \\
\hline$b_{f}$ & 0.18 \\
$T$ & 6 \\
$k_{i},(i=1,2)$ & $6.5,1.1$ \\
$\iota_{i},(i=1,2,3)$ & $0.1,0.1,0.1$ \\
$S_{i},(i=1,2,3)$ & $1.5,1.5,1.5$ \\
$S_{\theta}$ & 1.5 \\
$\boldsymbol{\Gamma}_{1}$ & $\boldsymbol{I}_{89 \times 89}$ \\
$\boldsymbol{\Gamma}_{2}$ & $2 \boldsymbol{I}_{89 \times 89}$ \\
$\sigma_{i},(i=1,2,3)$ & $0.03,0.08,0.08$ \\
$\rho_{1}$ & 1 \\
$r_{1}$ & 0.001 \\
$c_{i},(i=1,2,3)$ & 0.1 \\
$k_{a}$ & 6.5 \\
$\tau_{2}$ & 0.03 \\
\hline
\end{tabular}




$$
\begin{gathered}
\boldsymbol{J}(\boldsymbol{\beta})=\operatorname{diag}\left(\sin \left(0.5 \beta_{1} t\right)+2, \sin \left(0.5 \beta_{2} t\right)+2\right. \\
\left.\sin \left(0.5 \beta_{3} t+2\right)\right) \\
\boldsymbol{M}(\boldsymbol{\beta}, \dot{\boldsymbol{\beta}})=\operatorname{diag}\left(\cos \left(0.5 \beta_{1}+\dot{\beta}_{1}\right)+2, \cos \left(0.5 \beta_{2}\right.\right. \\
\left.\left.+\dot{\beta}_{2}\right)+2, \cos \left(0.5 \beta_{3}+\dot{\beta}_{3}\right)+2\right) \\
\boldsymbol{Q}(\boldsymbol{\beta}, \dot{\boldsymbol{\beta}})=\operatorname{diag}\left(\cos \left(0.5 \beta_{1}+\dot{\beta}_{1}\right) / 3+1, \cos \left(0.5 \beta_{2}\right.\right. \\
\left.\left.+\dot{\beta}_{2}\right) / 3+1, \cos \left(0.5 \beta_{3}+\dot{\beta}_{3}\right) / 3+1\right) \\
\boldsymbol{D}_{1}=\left[0.5 \cos \left(0.5 \beta_{1} t\right)+\sin (0.05 t) ;\right. \\
0.5 \cos \left(0.5 \beta_{2} t\right)+\sin \left(0.05\left(t+\frac{2 \pi}{3}\right)\right) \\
\left.0.5 \cos \left(0.5 \beta_{3} t\right)+\sin (0.05(t+4 \pi / 3)) ;\right] \\
\boldsymbol{D}_{2}=\left[0.5 \cos \left(0.5 \beta_{1} t\right)+3 \sin \left(0.5 \dot{\beta}_{1}\right)+\sin (0.05 t) ;\right. \\
0.5 \cos \left(0.5 \beta_{2} t\right)+3 \sin \left(0.5 \dot{\beta}_{2}\right)+\sin \left(0.05\left(t+\frac{2 \pi}{3}\right)\right) ; \\
\left.0.5 \cos \left(0.5 \beta_{3} t\right)+3 \sin \left(0.5 \dot{\beta}_{3}\right)+\sin (0.05(t+4 \pi / 3)) ;\right]
\end{gathered}
$$

In this simulation, three scenarios are set to verify the merits of the proposed method:

Scenario 1: Step change of the reference pitch angle happens at $t=10 \mathrm{~s}$. In this transient process, the user-defined time $T$ is selected as $1 \mathrm{~s}, 2 \mathrm{~s}$, and 3s, respectively.

Scenario 2: Influence of parameter change. Specifically, the value of the parameter matrix $\boldsymbol{J}(\boldsymbol{\beta})$ became half of the original value at $t=40 \mathrm{~s}$, which is shown in Fig. 5.

Scenario 3: Influences of disturbances. The disturbance matrices $\boldsymbol{D}_{1}$ and $\boldsymbol{D}_{2}$, which are shown in Fig. 6, are applied to the system at time $t=70 \mathrm{~s}$;

In scenario 1 , to verify that the proposed controller can tracking signal within a finite time, we chosen different settling time for the controller, which are $1 \mathrm{~s}, 2 \mathrm{~s}$, and $3 \mathrm{~s}$, respectively. Besides, the convergence rate function $\kappa(t)=$ $e^{t}$ is chosen in the design. We assume the reference pitch angle as $15^{\circ}$ with a step change to $20^{\circ}$ at $\mathrm{t}=10 \mathrm{~s}$. Noting that $\boldsymbol{y}_{d}$ is bounded in this study as beforementioned in Assumption 1, smooth processing on the stepping change signal has been conducted. Fig. 4 shows the tracking performance of the pitch angle $\beta_{1}$ in scenario 1 . As can be observed in this figure, before step change in reference pitch angle, both three controllers can ensure that the pitch angle $\beta_{1}$ can track the reference $\beta_{1}^{*}$ closely, with a tracking error of nearly 0 . At $\mathrm{t}=10 \mathrm{~s}$, the reference pitch angle suddenly steps from $15^{\circ}$ to $20^{\circ}$, and it is observed that the pitch angle $\beta_{1}$ can track the reference signal within the predefined times. Moreover, the transient performance of the controller improved effectively by choosing a shorter settling time $T$ properly. This keeps pace with the theoretical analysis.

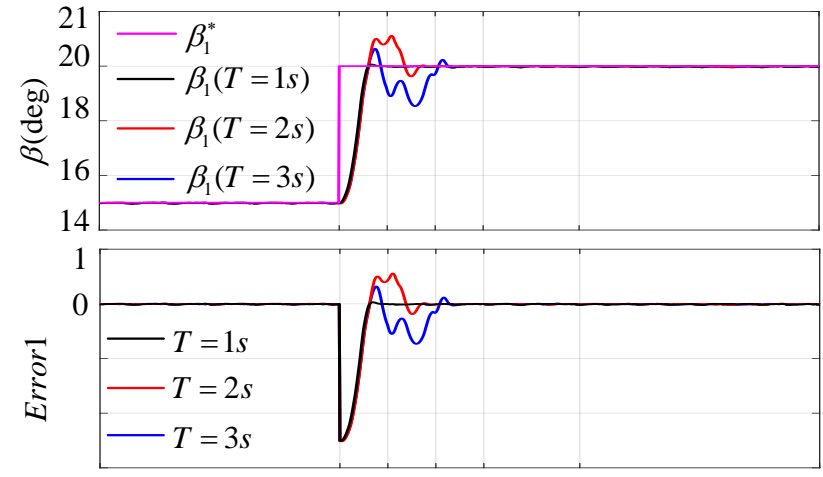

Time(s)

Fig. 4. A stepping change of the reference pitch angle

In scenario 2, the tracking performance of the proposed controller for blade 1 (the other two blades shares the same pitch angle adjustment mechanism and not shown here) under the influence of system parameter change is depicted in Fig. 5. Meanwhile, a traditional PID control method was introduced for comparison. As shown in Fig. 5, before the value of the parameter matrix $\boldsymbol{J}(\boldsymbol{\beta})$ is changed, the pitch angle $\beta_{1}$ can track the reference angle $\beta_{1}^{*}$ closely, and the tracking error is nearly 0 . Meanwhile, the tracking performance under the traditional PID control is relatively poor. From Fig. 5, it is readily known that the RBFNN based controller enjoys a faster error convergence and better rejection capability on parameter change effects compared to the traditional PID controller. After the parameter matrix $\boldsymbol{J}(\boldsymbol{\beta})$ suddenly changed, it is seen that the pitch angle $\beta_{1}$ can still track $\beta_{1}^{*}$ closely. The tracking error still remains at around 0 . This verifies the declaration that the proposed controller can address the control problems related to parameter changes.

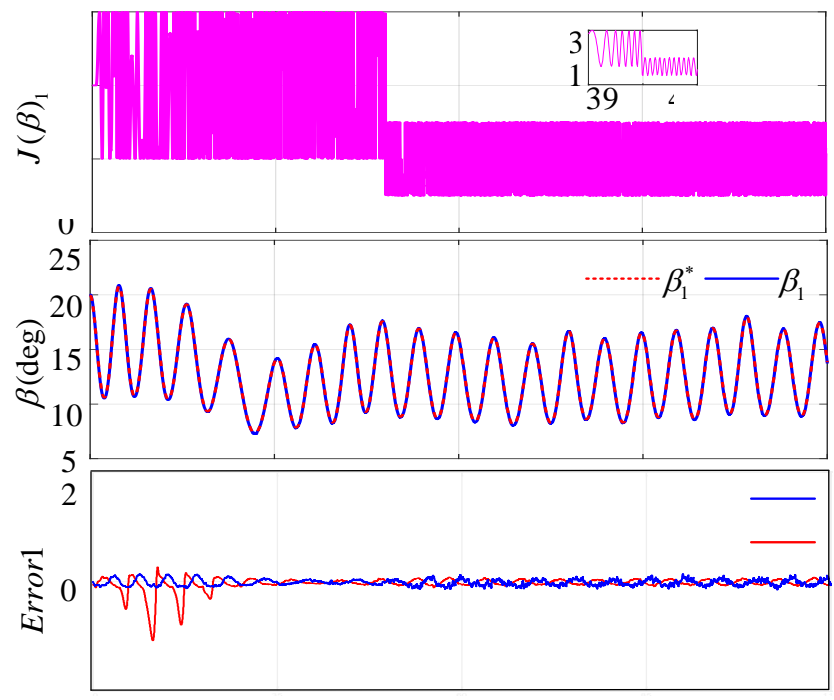

Time(s)

Fig. 5. The pitch angle tracking error of blade1 the parameters change on the system.

In scenario 3, the pitch angle tracking performance for blade 1 is depicted in Fig. 6. A traditional PID controller is also introduced for comparison. As can be observed, before 
disturbances $\boldsymbol{D}_{1}$ and $\boldsymbol{D}_{2}$ are introd uced into the system, the proposed controller ensure that the pitch angle $\beta_{1}$ can track the reference $\beta_{1}^{*}$ closely, with a tracking error of nearly 0 . The tracking error under the traditional PID controller is relatively greater, especially in the first 25 s of simulation. After adding the disturbances at $t=70 \mathrm{~s}$, it is evidenced that the tracking performance of pitch angle 1 is not heavily affected: the tracking error remains at around 0. Compared with the traditional PID controller, the fluctuation range of the RBFNN controller error is smaller. This indicates that the proposed IPC method can reject the disturbances and avoid their influences on tracking performance.

Hence, the validity of the proposed IPC method is verified.

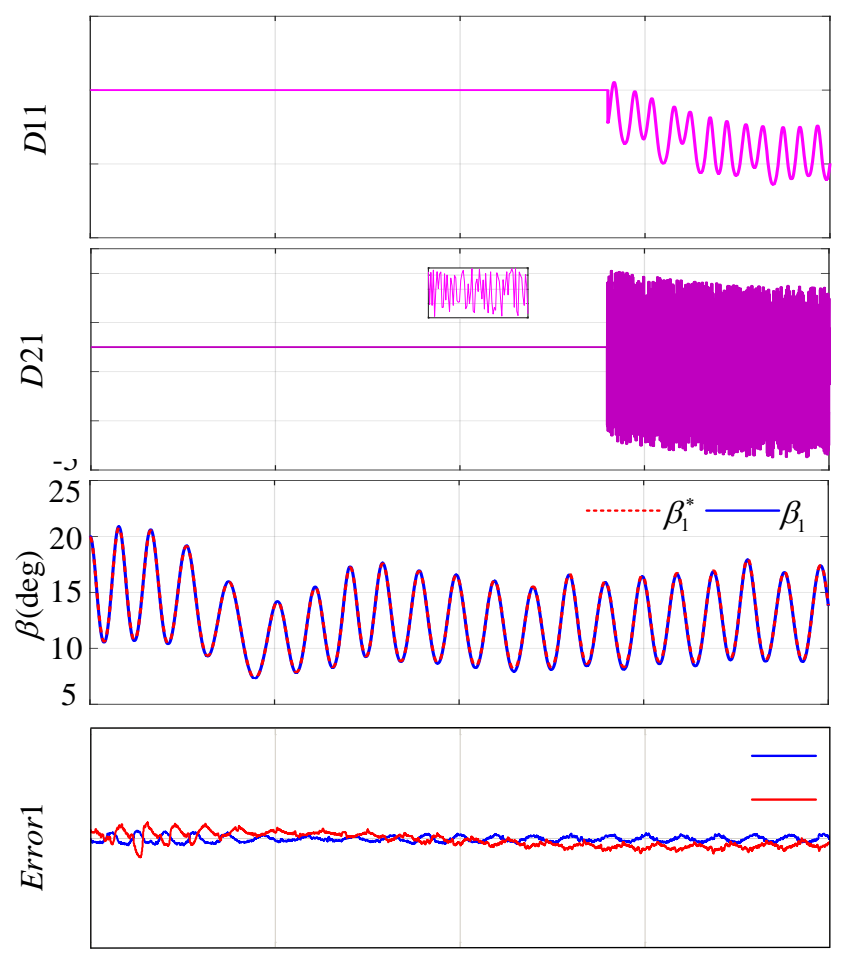

Time(s)

Fig. 6. The pitch angle tracking error of blade1 under system disturbances $\boldsymbol{D}_{1}$ and $\boldsymbol{D}_{2}$

\section{Conclusion}

In this paper, a neuro-adaptive based individual pitch tracking controller for offshore floating WTs is proposed to regulate the power output and mitigate dynamic loads. The neural- adaptive tracking controller designed in this study can track the desired pitch angle precisely and quickly, and can ensure that all the internal signals is bounded. The controller does not depend on the parameters of the system model, so there is no need to install additional sensors to measure the uncertain time-varying parameters of the system, which is economical. Compared with the traditional PID controller, under system parameter uncertainty and disturbances, this control method can achieve zero error tracking within a predefined finite time. Thus the performance of pitch angle regulation is guaranteed. On this basis, one can conclude that the superior pitch tracking ability realized in this work can ensure that the floating WTs can produce rated power output and mitigate loads in the presence of and disturbances.

\section{Acknowledgments}

The authors gratefully acknowledge the support provided by the National Natural Science Foundation of China (NO.51875058); Chongqing Basic Science and Frontier Technology Research Special (NO.CSTC2018jcyjAX0414; Chongqing Municipal Education Commission Science and Technology Research Project (NO.KJQN20180118) and Central University Frontier Discipline Special Project (NO.2019CDQYZDH025).

\section{References}

[1] Chatterjee, S., Chatterjee, S., et al: 'Review on the techno-commercial aspects of wind energy conversion system', IET Renewable Power Generation, 2018, 12, (14), pp. 1581-1608.

[2] Ohlenforst, K., Sawyer, S., Dutton, A., et al: 'Global wind report 2018', Global Wind Energy Council, Brussels, Belgium, Tech. Rep., 2019.

[3] Zountouridou, E.I., Kiokes, G.C., Chakalis, S., et al: 'Offshore floating wind parks in the deep waters of Mediterranean Sea', Renewable and Sustainable Energy Reviews, 2015, 51, pp. 433-448.

[4] Zhang, Y., Chen, Z., Cheng, M.: 'Proportional resonant individual pitch control for mitigation of wind turbines loads', IET Renewable Power Generation, 2013, 7, (3), pp. 191-200.

[5] Han, B., Zhou, L., Yang F., et al: 'Individual pitch controller based on fuzzy logic control for wind turbine load mitigation', IET Renewable power generation, 2016, 10, (5), pp. 687-693.

[6] Zhang, Y., Cheng, M., Chen, Z.: 'Load mitigation of unbalanced wind turbines using PI-R individual pitch control', IET Renewable Power Generation, 2014, 9, (3) pp. 262-271.

[7] Hoghooghi, H., Chokani, N., Abhari,R.S.: 'Effectiveness of individual pitch control on a $5 \mathrm{MW}$ downwind turbine’, Renewable Energy, 2019, 139, pp. 435-446.

[8] Wright, A.D., Fingersh, L.J.: 'Advanced control design for wind turbines', National Renewable Energy Lab, 2008, pp. 19-27.

[9] Stol, K., Balas, M.: 'Periodic disturbance accommodating control for blade load mitigation in wind turbines’, J. Solar Energy Eng., 2003, 125, (4), pp. 379385.

[10] Odgaard, P.F., Stoustrup, J., Kinnaert, M.: 'Faulttolerant control of wind turbines: A benchmark model', IEEE Trans. Control Syst. Technol., 2013, 21, (4), pp. 1168-1182.

[11] Jelavic, M., Petrovic, V., Peric, N.: 'Individual pitch control of wind turbine based on loads estimation', IEEE Proc. Industrial and Electronics., Florida, America, 2008, pp. 228-234.

[12] Yuan, Y., Chen, X., Tang, J.: 'Multivariable robust blade pitch control design to reject periodic loads on wind turbines', Renewable Energy, 2020, 146, pp. 329-341.

[13]Dai, J.C., Hu, Y.P., Liu, D.S.: 'Modeling and characteristics analysis of the pitch system of large scale wind turbine', Journal of Mechanical Engineering Science-IMechE, 2011, 225, (3), pp. 558-567. 
[14] Chen, J., Song, Y.D.: 'Dynamic loads of variable-speed wind energy conversion system', IEEE Trans. Ind. Electron., 2015, 63, (1), pp. 178-188.

[15] Chen, Z.J., Stol, KA, Mace, BR: 'Wind turbine blade optimization with individual pitch and trailing edge flap control', Renewable Energy, 2017, 103, pp. 750-765.

[16] Asl, H.J., Yoon, J.: 'Power capture optimization of variable-speed wind turbines using an output feedback controller', Renew Energy, 2016, 88, pp. 517-525.

[17] Abdelbaky, M.A., Liu, X.J., Jiang, D.: 'Design and implementation of partial offline fuzzy model-predictive pitch controller for large-scale wind-turbines', Renewable Energy, 2020, 145, pp. 981-996.

[18] Yuan, Y., Tang, J.: 'Adaptive pitch control of wind turbine for load mitigation under structural uncertainties', Renewable Energy, 2017, 105, pp. 483494.

[19]Her, S., Huh, J., Kim, B.: 'Formula for estimating the uncertainty of manufacturer's power curve in pitchcontrolled wind turbines', IET Renewable Power Generation, 2018, 12, (3), pp. 292-297.

[20] Dubois, A., Leong, Z.Q., Nguyen, H.D., et al: 'Uncertainty estimation of a CFD-methodology for the performance analysis of a collective and cyclic pitch propeller', Applied Ocean Research, 2019, 85, pp. 73-87.

[21] Prasad, S., Purwar, S., Kishor, N.: 'Non-linear sliding mode control for frequency regulation with variablespeed wind turbine systems', International Journal of Electrical Power \& Energy Systems, 2019, 107, pp. 1933.

[22] Imran, R.M., Hussain, D.M.A., Soltani, M., et al: 'Optimal tuning of multi variable disturbance-observerbased control for flicker mitigation using individual pitch control of wind turbine', IET Renewable Power Generation, 2017, 11, (8), pp. 1121-1128.

[23] Navalkar, S.T., Van Solingen, E., Van Wingerden, J.: 'Wind Tunnel Testing of Subspace Predictive Repetitive Control for Variable Pitch Wind Turbines', IEEE Transactions on Control Systems Technology, 2015, 23, (6), pp. 2101-2116.

[24] Civelek, Z., Lüy, M., Çam, E., et al: 'A new fuzzy logic proportional controller approach applied to individual pitch angle for wind turbine load mitigation', Renewable Energy, 2017, 111, pp. 708-711.

[25] Poultangari, I., Shahnazi, R., Sheikhan, M.: 'RBF neural network based PI pitch controller for a class of 5-MW wind turbines using particle swarm optimization algorithm’, ISA Transactions, 2012, 51, pp. 641-648.

[26] Mirzaei, M., Soltani, M., Poulsen, N.K.: 'An MPC approach to individual pitch control of wind turbines using uncertain LIDAR measurements', European Control Conference. IEEE., 2013.

[27]Wu, Y., Lee, C., Chen, C., et al: 'Optimization of the Wind Turbine Layout and Transmission System Planning for a Large-Scale Offshore WindFarm by AI Technology', IEEE Transactions on Industry Applications, 2014, 50, (3), pp. 2071-2080.

[28] Heier, S., Roth, G., Waddington, R.: 'Grid integration of wind energy : onshore and offshore conversion systems', John Wiley \& Sons, 2014.

[29] Imai, A.K., Costa R.R., Hsu L., et al: 'Multivariable adaptive control using high-frequency gain matrix factorization', Automatic Control IEEE Transactions on, 2004, 49, (7), pp: 1152-1156.

[30]Zhao, K., Song, Y.D., Ma, T.D., et al: 'Prescribed performance control of uncertain Euler-Lagrange systems subject to full-state constraints', IEEE transactions on neural networds and learning systems, 2018, 29, (8).

[31]Zhao, K., Song, Y.D., Ma, TD, He, L.: 'Prescribed performance control of uncertain Euler-Lagrange systems subject to full-state constraints', IEEE transactions on neural networds and learning systems, 2018, 29, (8).

[32] Chen, L., Wang, Q.: 'Adaptive robust tolerant control for a class of uncertain MIMO non-affine non-linear systems', IEEE/CAA Journal of Automatica Sinica (JAS), 2016, 3, (1), pp. 105-112.

[33]Liu, Y.J., Tong, S.: 'Barrier Lyapunov function-based adaptive control for a class of non-linear pure-feedback systems with full state constraints', Auomatica, 2016, 64, pp. 70-75.

[34] Imai, A.K., Costa, R.R., Hsu, L., Tao, G., Kokotovic, P. V.: 'Multivarialbe adaptive control using high-frequency gain matrix factorization', IEEE Trans. Autom. Control., 2004, 49, (7), pp. 1152-1157. 